\title{
APLICAÇÕES DOS MODELOS DE ELEVAÇÃO SRTM EM GEOMORFOLOGIA
}

\section{SRTM DEMS APPLICATIONS IN GEOMORPHOLOGY}

\author{
Carlos Henrique Grohmann \\ Instituto de Geociências - USP - Rua do Lago, 562 - CEP 05508-080 - São Paulo, SP \\ guano@usp.br, carlos.grohmann@gmail.com \\ Claudio Riccomini \\ Instituto de Geociências - USP \\ riccomin@usp.br \\ Samar dos Santos Steiner \\ Instituto de Geociências - USP \\ samar.steiner@gmail.com
}

\section{RESUMO}

A missão Shuttle Radar Topography Mission (SRTM) foi realizada para mapear o relevo da área continental da Terra com interferometria de radar entre $60^{\circ}$ de latitude norte e $54^{\circ}$ de latitude sul. A utilização de Modelos Digitais de Elevação (MDEs) em geomorfologia permite o cálculo de variáveis associadas ao relevo com rapidez e precisão. Neste artigo, apresenta-se uma visão geral de aplicações dos modelos de elevação SRTM em análises geomorfológicas realizadas em território brasileiro, bem como uma apreciação das limitações existentes e de perspectivas futuras.

Palavras-chave: SRTM, geomorfologia, neotectônica, morfotectônica.

\begin{abstract}
The Shuttle Radar Topography Mission (SRTM) was realized to map the morphology of the Earth's continental areas between $60^{\circ} \mathrm{N}$ and $54^{\circ} \mathrm{S}$, using radar interferometry. Application of Digital Elevation Models (DEMs) in geomorphology allow calculation of topography-related variables with speed and precision. In this paper, we present a general view of geomorphological studies supported by SRTM data, conducted within the Brazilian territory, as well as an appreciation of its limitations and future perspectives.
\end{abstract}

Keywords: SRTM, geomorphology, neotectonics, morphotectonics.

\section{INTRODUÇÃO}

A utilização de Modelos Digitais de Elevação (MDEs) em geomorfologia permite o cálculo de variáveis associadas ao relevo com rapidez e precisão. O crescimento da capacidade de processamento e armazenamento de dados dos computadores e a evolução dos Sistemas de Informação Geográfica (SIGs) encontra-se em um estágio no qual é possível trabalhar em extensas áreas com alta resolução espacial, sem perda significativa do tempo de processamento envolvido.

Neste artigo, apresenta-se uma visão geral de aplicações dos modelos de elevação SRTM em análises geomorfológicas, bem como uma apreciação das limitações existentes e de perspectivas futuras. 


\section{MODELOS DE ELEVAÇÃO SRTM}

A missão Shuttle Radar Topography Mission (SRTM - Farr e Kobrick, 2000; van Zyl, 2001; Rabus et al., 2003; Farr et al., 2007) foi realizada em conjunto pela agência espacial norte-americana (National Aeronautics and Space Administration - NASA), a National Geospatial-Intellingence Agency (NGA), o Departamento de Defesa dos Estados Unidos (DoD) e as agências espaciais alemã (Deustches Zentrum für Luft- und Raumfahrt - DLG) e italiana (Agenzia Spaziale Italiana - ASI) em fevereiro de 2000, para mapear o relevo da área continental da Terra com interferometria de radar de abertura sintética (InSAR), entre $60^{\circ}$ de latitude norte e $54^{\circ}$ de latitude sul, o que corresponde à aproximadamente $80 \%$ das áreas emersas do planeta.

Os princípios da InSAR foram desenvolvidos no início dos anos 1980 (Zebker e Goldstein, 1986). A altitude de um ponto na superfície terrestre pode ser determinada a partir da diferença de fase entre reflexões de um mesmo sinal de radar captado por dois receptores distantes um do outro (Rabus et al., 2003).

No caso da missão SRTM, os canais principais de transmissão e recepção para as bandas C e X foram posicionados na área de carga do ônibus espacial Endeavour, enquanto que as antenas secundárias (somente recepção) foram colocadas na extremidade de um mastro retrátil de $60 \mathrm{~m}$ de comprimento. Para uma descrição detalhada de interferometria de radar de visada lateral, consultar, por exemplo, Bamler e Hartl (1998).

Os dados brutos foram processados pela NASA e, apesar de obtidos com resolução espacial de 1 segundo de arco (aproximadamente 30 m no equador), estão disponíveis com este nível de detalhe apenas para a área dos Estados Unidos. Para os outros países, houve uma reamostragem dos dados para 3 segundos de arco (aproximadamente $90 \mathrm{~m}$ ) de resolução espacial. O produto final possui precisão vertical global de $\pm 16 \mathrm{~m}$ e horizontal de $\pm 20 \mathrm{~m}$ (Rabus et al., 2003). Para a América do Sul, a precisão vertical é de $6,2 \mathrm{~m}$ e a horizontal de $9,0 \mathrm{~m}$ (Rodriguez et al., 2006). O produto é georreferenciado ao datum WGS84 em coordenadas geográficas decimais e pode ser obtido via Internet. Os dados para o território brasileiro processados pela Embrapa (Miranda, 2005) estão disponíveis segundo a articulação das folhas topográficas em escala 1:250.000.

Deve-se levar em conta que o resultado das técnicas empregadas na missão SRTM é a geração de Modelos Digitais de Elevação (MDEs), pois os sinais de radar são refletidos, por exemplo, pelo dossel das árvores em áreas densamente florestadas e não pelo terreno subjacente. Os termos Modelo Digital de Terreno (MDT) e Modelo Numérico de Terreno (MNT) devem ser reservados para casos onde o modelo é produzido a partir de valores de altitude do nível do solo, obtidos, por exemplo, em mapas topográficos (curvas de nível), levantamentos por GPS ou por altimetria a laser(LiDAR).

Uma característica importante a ser observada é a metodologia empregada na reamostragem dos dados originais para 3 segundos de arco. Os dados distribuídos pelo Serviço Geológico dos Estado Unidos (USGS) foram gerados a partir da média dos valores originais, em uma janela de 3x3 pixels, 0 que resulta em um modelo suavizado com relação ao original. Os dados disponibilizados pela NASA [2] foram gerados a partir do valor do pixel central da janela $3 \times 3$ e portanto possuem informações dos dados originais (Figura 1). 


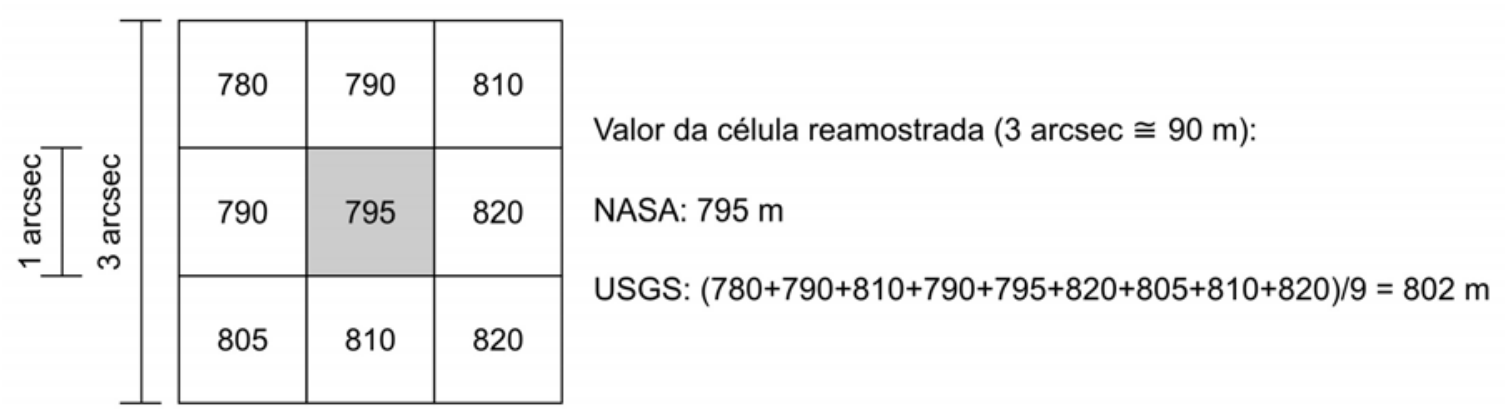

Figura 1 - Métodos de reamostragem dos dados SRTM utilizados pela NASA e pelo USGS, para geração dos modelos com resolução de 3 segundos de arco (adaptado de Steiner, 2007).

Os modelos SRTM30_PLUS (Becker e Sandwell, 2007) consistem em um modelo global com resolução espacial de 30 segundos de arco (aproximadamente $1 \mathrm{~km}$ ), criado a partir da fusão de dados de diversos projetos.

Nas áreas continentais, o modelo utiliza principalmente os dados do USGS SRTM30, uma reamostragem para $1 \mathrm{~km}$ dos dados SRTM originais. Para as áreas em altas latitudes, onde não há dados SRTM, foi utilizado o modelo GTOPO30 . A área oceânica é baseada no modelo global de Smith e Sandwell (1997), com resolução espacial de 1 minuto, entre as latitude $\pm 72^{\circ}$. Modelos de mais alta resolução foram incorporados a partir dos projetos LDEO Ridge Multibeam Synthesis Project e NGDC Coastal Relief Model. A batimetria do ártico deriva da International Bathymetric Chart of the Oceans (Jakobsson et al., 2000).

A resolução de aproximadamente $90 \mathrm{~m}$ dos dados SRTM disponíveis pode ser considerada razoável para análises em escalas pequenas ou médias, mas não pode ser aplicada em estudos de detalhe. Uma alternativa viável consiste em interpolar os dados originais com uma resolução maior (ou seja, para obter células de tamanho menor ao original). Esta abordagem não irá aumentar o nível de detalhe do modelo resultante, porém resultará em uma superfície com coerência de suas propriedades angulares (p.ex., declividade, orientação de vertentes etc) entre as células vizinhas, fator importante em análise morfométrica.

Valeriano et al. (2006), ao estudarem a morfometria de pequenas bacias de drenagem na Amazônia, propõem uma metodologia para a reamostragem dos modelos SRTM baseada na interpolação por krigagem. A krigagem é uma técnica geoestatística utilizada para interpolar o valor de uma variável (p.ex., elevação) em pontos não amostrados a partir dos valores de pontos amostrados próximos. A influência que cada valor original exerce sobre o valor interpolado é determinada pela função variograma. O gráfico do variograma (ou semi-variograma) para superfícies com variações suaves, tais como a superfície do nível freático ou da topografia, geralmente pode ser ajustado a um modelo Gaussiano, que apresenta uma região de baixa inclinação próxima da origem (Burrough, 1987).

A reamostragem dos dados de elevação de 3 segundos de arco para 1 segundo de arco se mostrou bastante eficaz (Figura 3), tanto em termos de análise e identificação visual das características do relevo, quanto na extração de parâmetros morfométricos, tais como declividade, orientação de vertentes e canais de drenagem. Deve-se levar em consideração que a variação de altura do dossel pode resultar tanto em suavização da superfície modelada, com conseqüente sub-estimação de parâmetros derivados, quanto em exagero da profundidade de canais de drenagem, e portanto é necessário cautela ao se trabalhar com esses modelos em áreas densamente florestadas (Valeriano et al., 2006). 


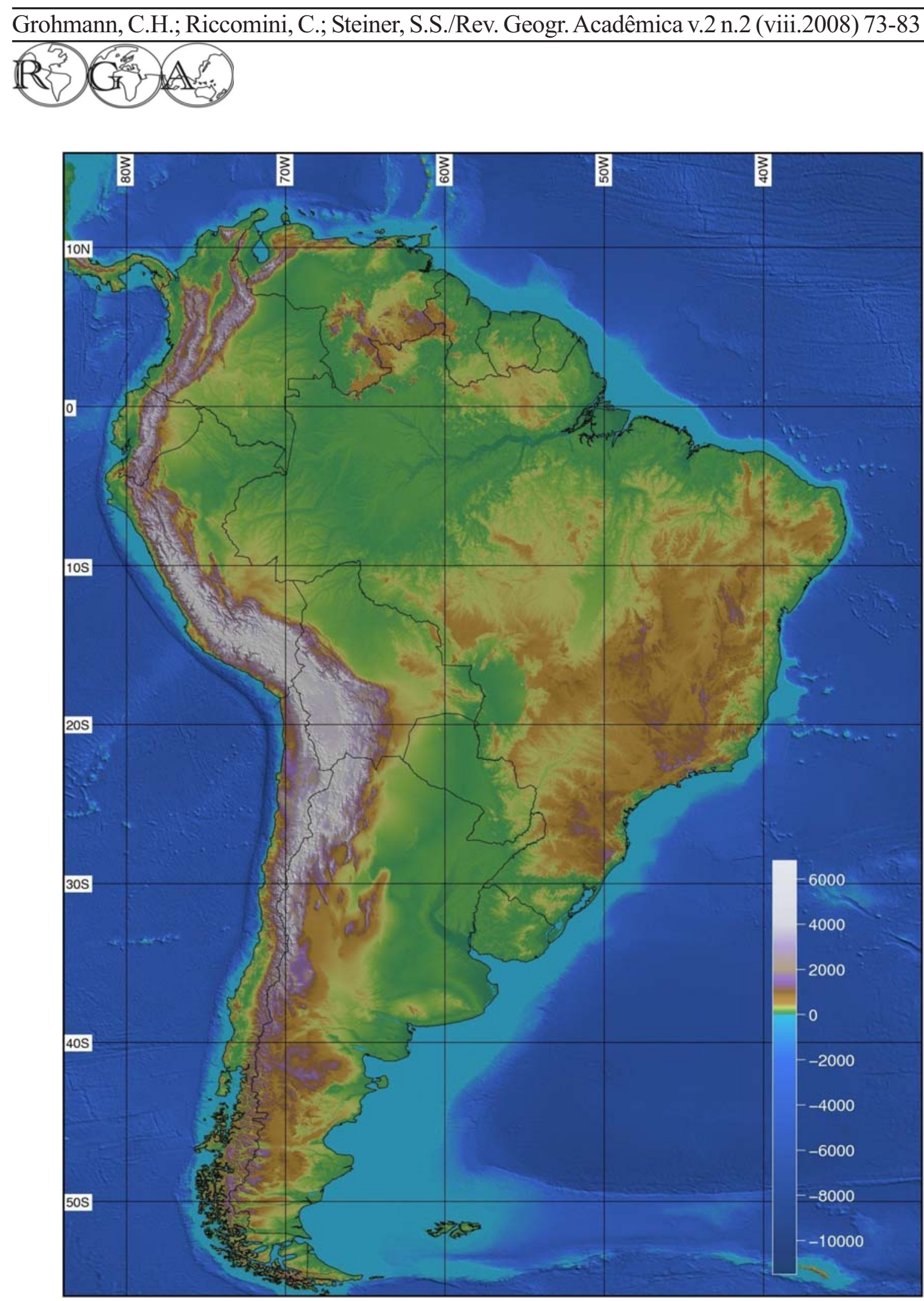

Figura 2 - Modelo SRTM30_PLUS para a América do Sul. (processamento: Carlos H. Grohmann) 

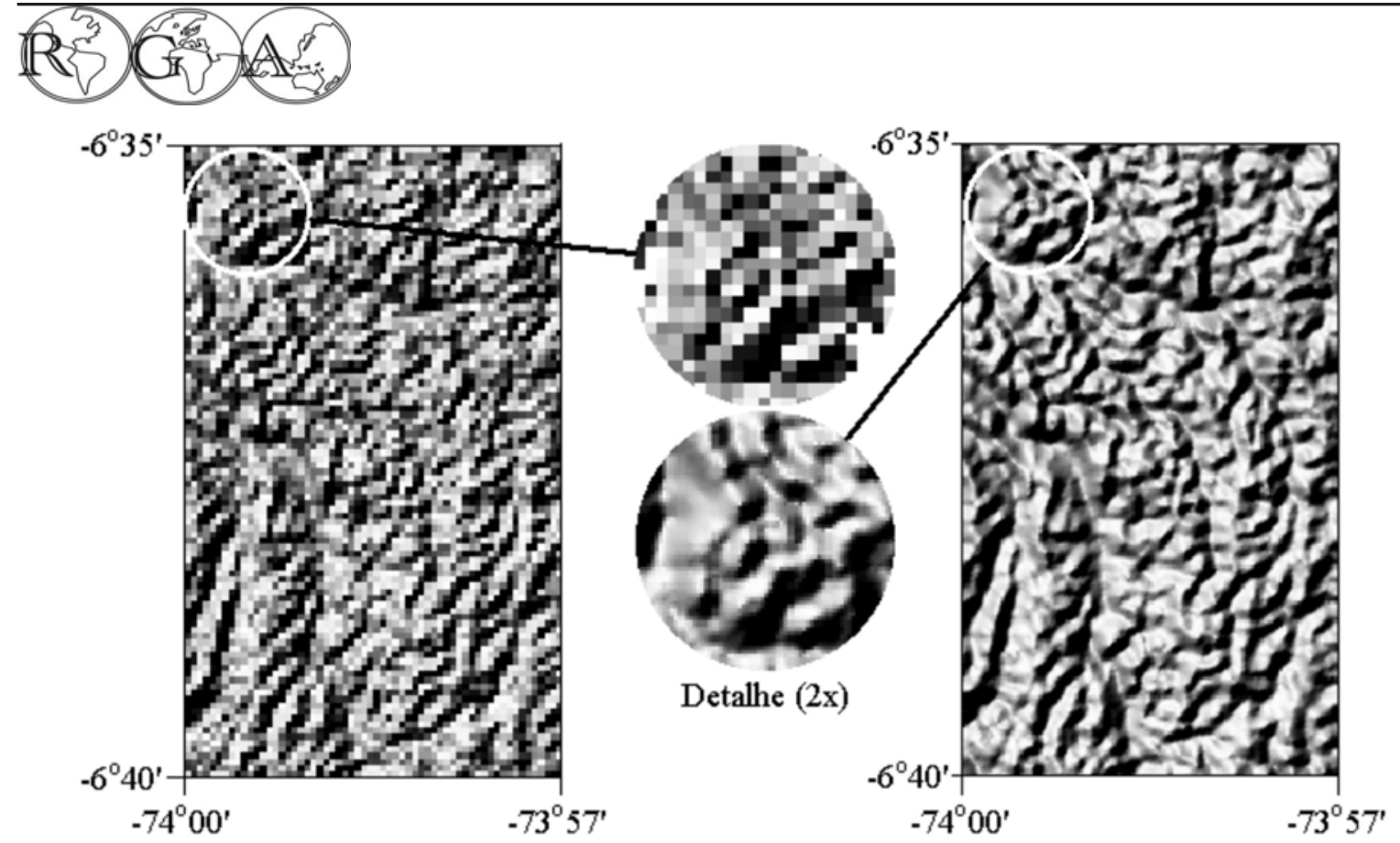

Escala

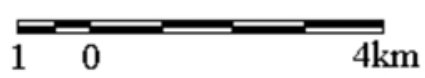

Figura 3 - Relevo sombreado de modelos de elevação SRTM originais (esq.) e reamostrados por krigagem (dir.). Figura 4.15 de Valeriano (2004).

Grohmann e Steiner (2008) propõe um refinamento do método de Valeriano et al. (2006), a fim de minimizar o efeito de suavização do relevo encontrado no modelos reamostrados. Uma vez que a superfície topográfica apresenta alta correlação espacial, o variograma é ajustado apenas em sua porção inicial (Figura 4). O cálculo da krigagem é limitado a um raio de busca compatível com um matriz de $3 \times 3$ ou $5 \times 5$ células dos dados originais, e o efeito pepita é determinado pela precisão vertical dos dados, sendo que para a América do Sul os modelos SRTM apresentam erro vertical absoluto de $\pm 9 \mathrm{~m}$ (Rodriguez, et al., 2006).

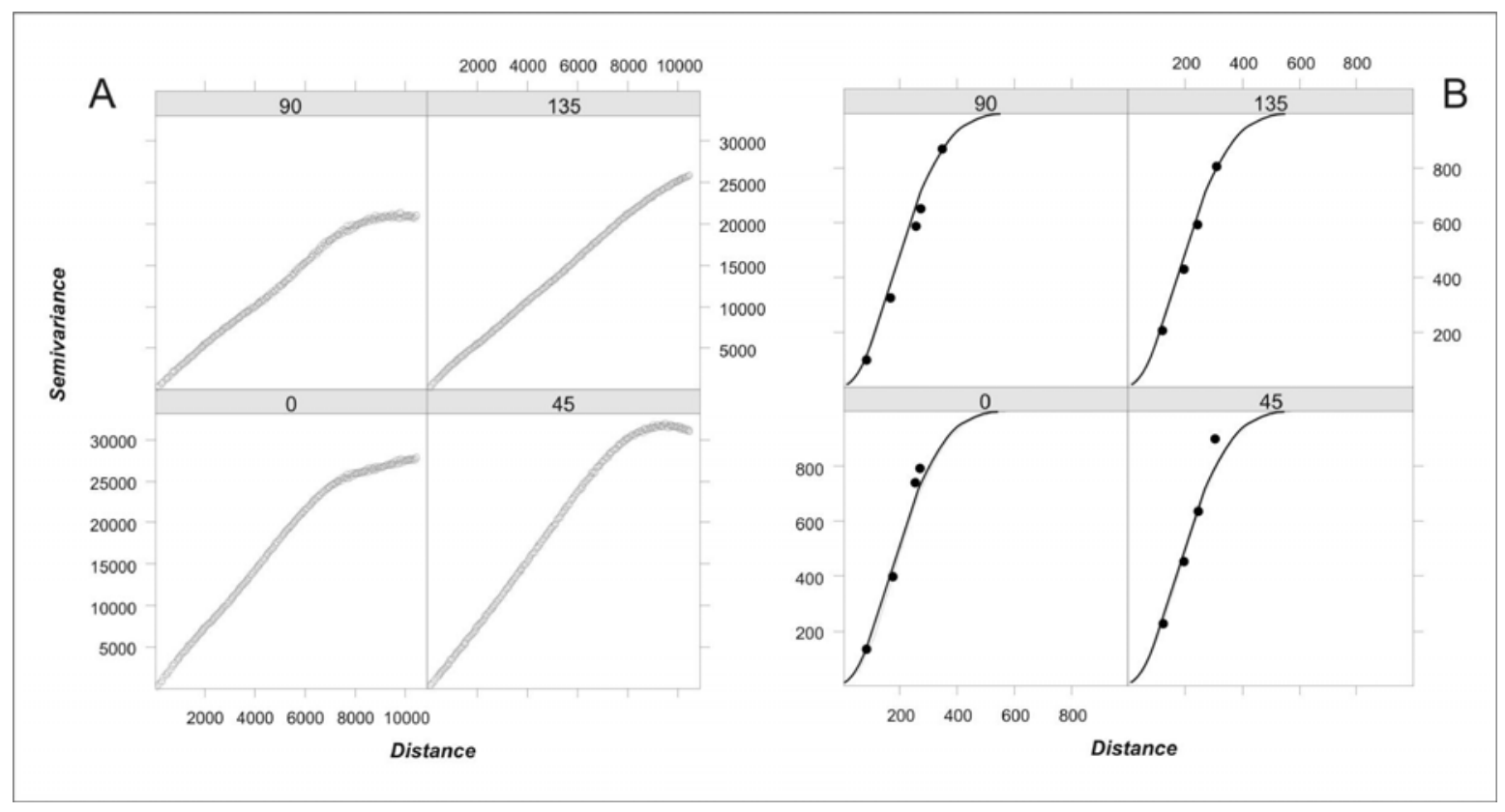

Figura 4 - Análise do variograma em quatro direções para a área da Serra do Japi (SP). A) Variogramas completos. B) Porção inicial do gráfico (Grohmann e Steiner, 2006). 


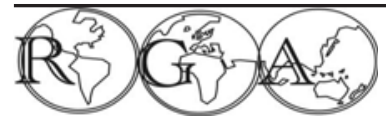

Os modelos SRTM apresentam áreas sem valores de elevação, chamadas de vazios (voids), decorrentes de problemas na reflexão dos sinais de radar em corpos d'água ou em vertentes com mergulho elevado (Farr e Kobrick, 2000; Farr et al., 2007). A interpolação dos dados com raio de busca pequeno (ou seja, utilizando poucos valores) significa que vazios maiores que a janela de busca não serão completamente "preenchidos". Por outro lado, utilizar um número maior de dados aumenta drasticamente o tempo de interpolação e resulta em superfícies muito suaves, sem o detalhamento desejado. No caso da presença de vazios muito grandes, uma alternativa viável é preencher os vazios antes da reamostragem.

O valor do efeito pepita, relacionado por Valeriano et al. (2006) ao erro vertical dos modelos, também irá influenciar a suavização da superfície reamostrada. Nos testes realizados, o valor de $10 \mathrm{~m}^{2}$ foi considerado suficiente para remover o ruído dos dados sem comprometer o detalhamento da topografia (Grohmann e Steiner, 2008).

\section{APLICAÇÕES}

Neste capítulo são apresentadas algumas aplicações dos modelos SRTM em análises geomorfológicas. Devido ao elevado número de publicações existentes, apenas trabalhos realizados em território brasileiro serão apresentados.

Carvalho e Latrubesse (2004), analisaram a potencialidade de uso dos modelos SRTM, na época ainda pouco difundidos entre pesquisadores brasileiros, no estudo da macrogeomorfologia da bacia do Rio Araguaia. Apesar dos produtos gerados se mostrarem eficazes na compartimentação do relevo, os autores apontam para problemas encontrados nos modelos em áreas de baixa declividade. Esses problemas são relacionados ao ruído aleatório inerente aos modelos SRTM e à deficiência da reflexão dos sinais de Radar em corpos d'água e em áreas alagadas (Farr e Kobrick, 2000; Guth, 2006; Farr et al., 2007).

Almeida Filho et al. (2005) estudaram a integração de dados SRTM com imagens orbitais multiespectrais do sensor ASTER (Advanced Spacebome Thermal Emission and Reflection Radiometer - Kahle et al., 1991), para o astroblema de Serra da Cangalha, localizado na divisa entre os estados de Tocantins e Maranhão (Figura 5). De acordo com os autores, o uso de imagens multiespectrais de alta resolução aliadas a modelos de elevação melhoram significativamente a interpretação estrutural de áreas geologicamente complexas.

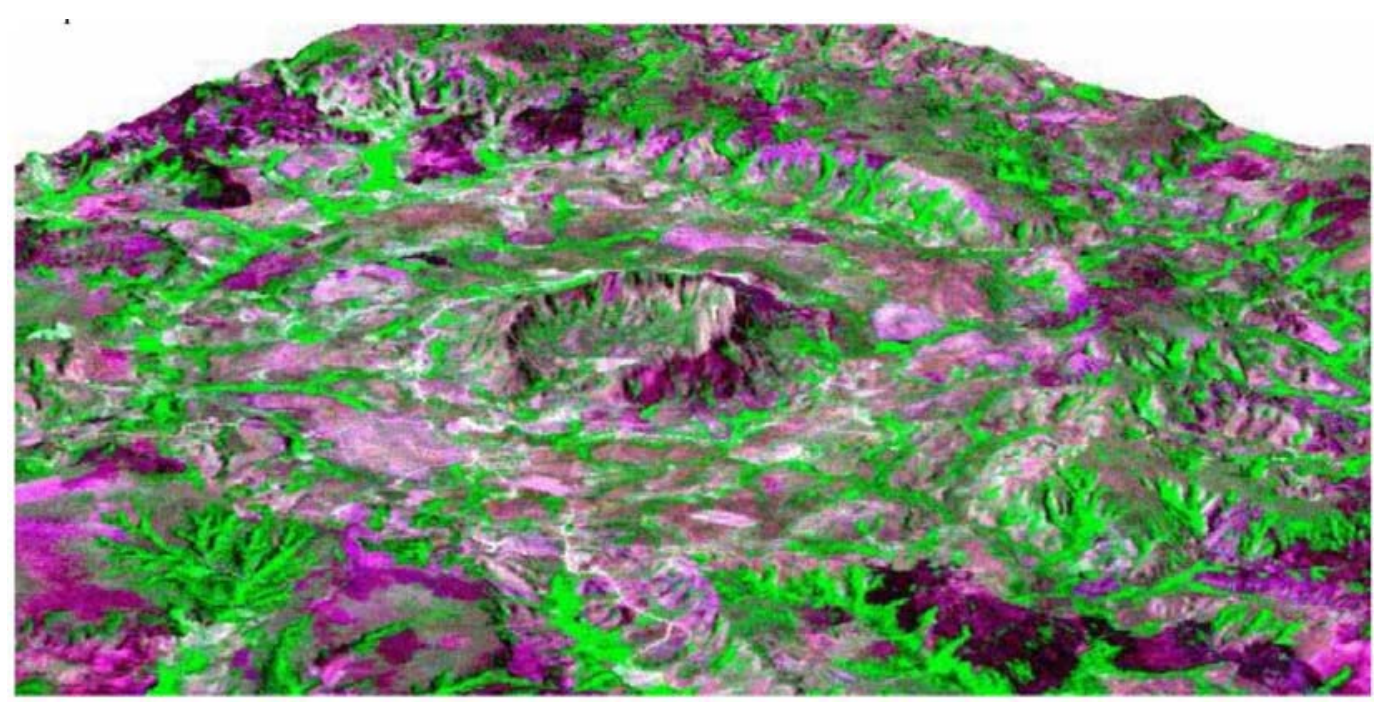

Figura 5 - Vista em perspectiva, de norte para sul, do astroblema de Serra da Cangalha, obtida através da sobreposição de composição falsa-cor ASTER (RGB=231) e modelo SRTM. O diâmetro do anel externo é de aproximadamente 14 km. Adaptado da figura 4 de Almeida Filho et al. (2005). 


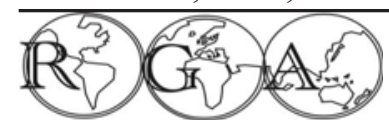

Grohmann et al. (2007) realizaram uma avaliação do uso dos modelos SRTM em geomorfologia e morfotectônica, na área do Maciço Alcalino de Poços de Caldas (MG). Foram considerados os parâmetros declividade, orientação de vertentes, rugosidade de relevo e superfícies de base. Como conclusão geral, verificou-se que os modelos SRTM possuem nível de detalhe suficiente para se trabalhar em escalas de até 1:50.000, sendo que, neste caso, deve-se reamostrar os dados para adequar a resolução à escala de semi-detalhe. Dentre os produtos derivados do modelo de elevação, destaca-se a derivação direta da rede de drenagem, com resultado bastante satisfatório comparada ao mapa topográfico (Figura 6). Para análises em escalas de 1:100.000 ou menores, até 1:500.000, a reamostragem dos dados não é explicitamente necessária, porém para estudos regionais o volume de dados torna-se demasiado denso para o detalhe exigido, sendo recomendável a reamostragem para a escala de trabalho.
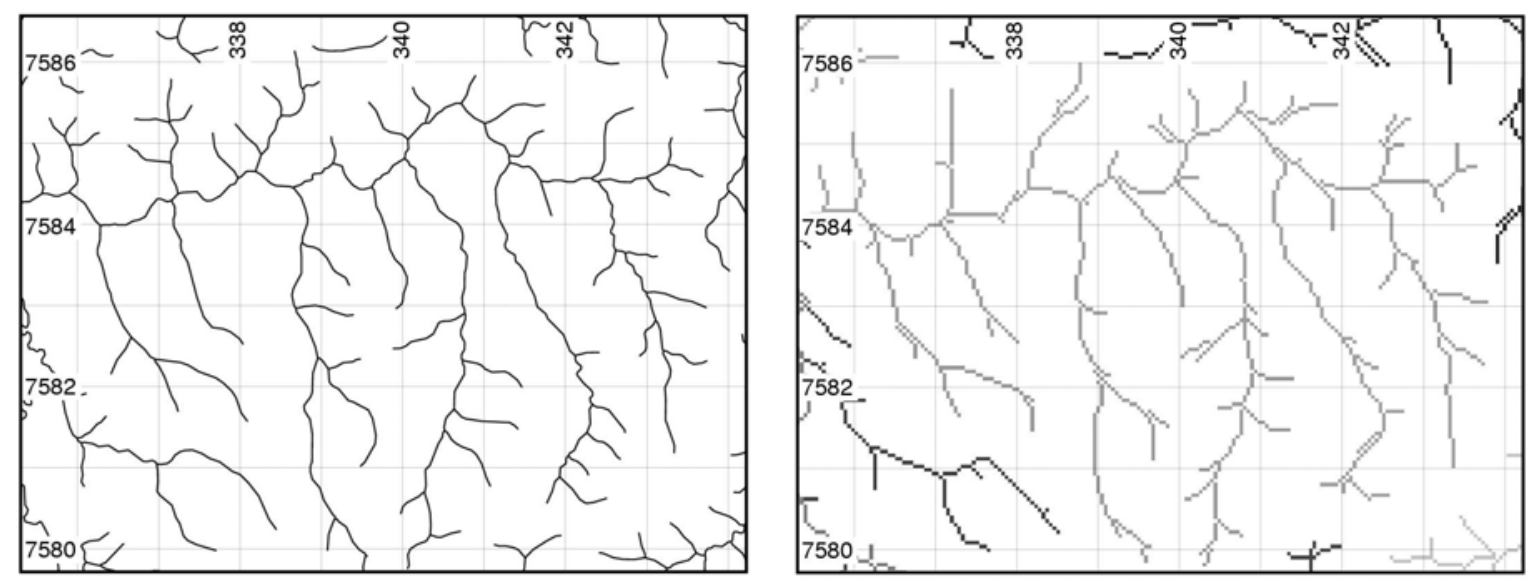

Figura 6 - Comparação entre drenagens do mapa topográfico 1:50.000 (esquerda) e extraídas automaticamente do modelo de elevação reamostrado para 50 m (direita). Adaptado de Grohmann et al. (2007).

Rossetti et al. (2007), utilizaram dados SRTM, Landsat 5-TM e Landsat 7-ETM+ no estudo da paleogeografia da Ilha de Marajó (PA). A análise das imagens de satélite, dos modelos de elevação e de dados sedimentológicos permitiu a delimitação de diversos paleocanais e o reconhecimento de um paleossistema estuarino pleistoceno, que teria evoluído a um sistema lacustre devido à atividade tectônica, responsável pela captura da drenagem do Rio Tocantins e conseqüente individualização da Ilha do Marajó, até então ligada ao continente.

A influência da neotectônica na configuração dos sistemas de drenagem da Bacia Amazônica também é apontada por Almeia-Filho e Miranda (2007). Uma ampla rede de drenagens, não observável em imagens orbitais ópticas ou de radar devido à cobertura vegetal, foi identificada em modelos SRTM (Figura 6). As análises indicam que o traçado atual do baixo Rio Negro é o resultado de uma megacaptura fluvial, controlada por estruturas NW-SE. O arranjo estrutural da área força a passagem do rio por um estreito, o que bloqueia parcialmente o fluxo e gera uma área mais larga à montante, semelhante $\mathrm{a}$ um lago. $\mathrm{O}$ ambiente de baixa energia favoreceu a deposição de sedimentos que formaram o arquipélago das Ilhas Anavilhanas, o maior conjunto de ilhas fluviais do mundo.

A possibilidade de identificar feições de relevo sutis, que seriam mascaradas pela densa cobertura vegetal da região Amazônica em imagens de sensoriamento remoto ótico, foi explorada por Ibanez et al. (2007). A disposição da rede de drenagem de uma área a leste de Manaus (AM) foi utilizada como indicador de padrões estruturais propícios à migração e/ou acumulação de hidrocarbonetos, como falhas e estruturas dômicas (Figura 7). Concluiu-se que análise dos dados morfoestruturais pode direcionar levantamentos geofísicos, e assim reduzir os riscos de campanhas prospectivas em áreas de fronteira exploratória, como as bacias sedimentares paleozóicas da Amazônia. 


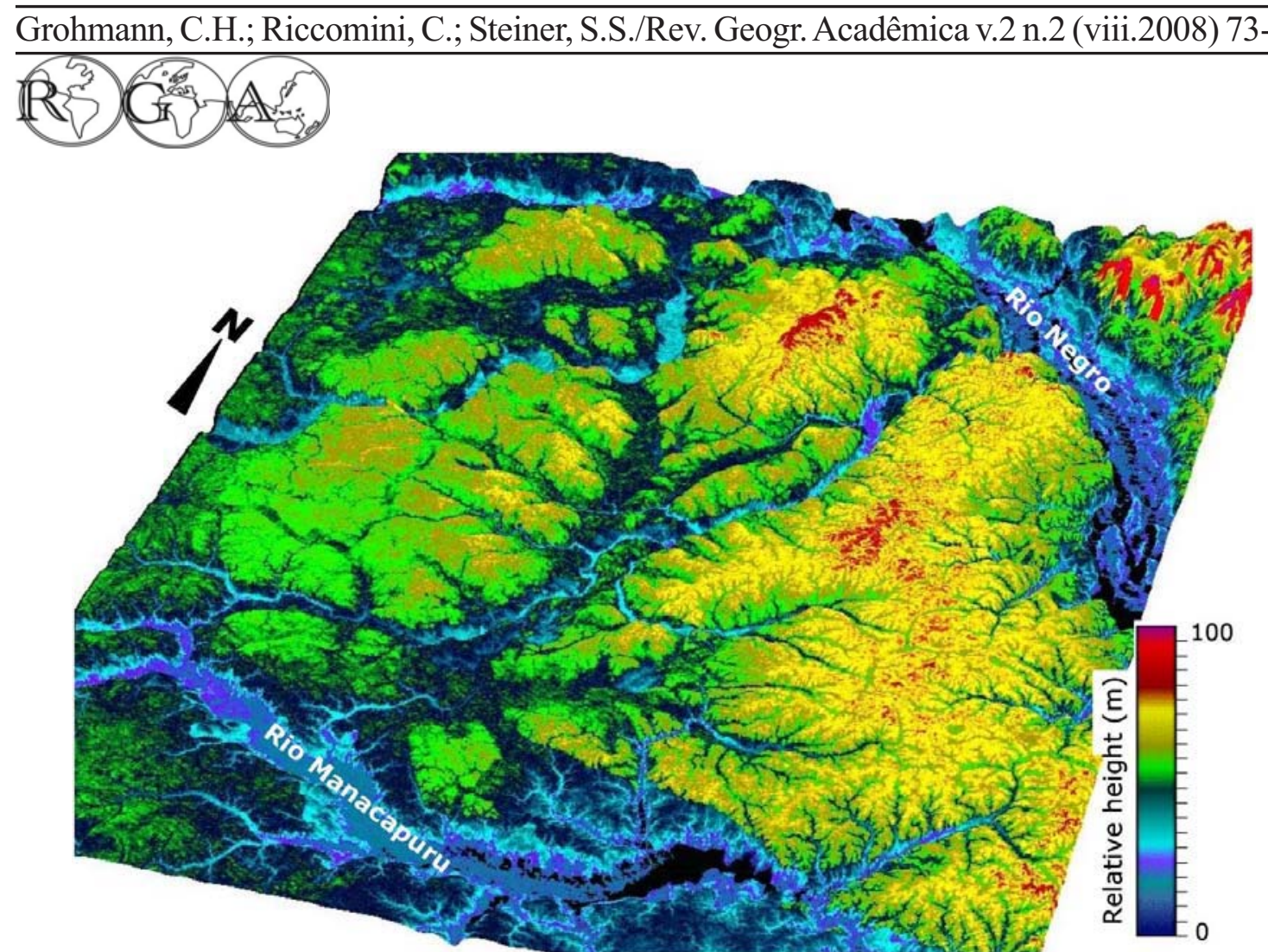

Figura 6 - Modelo SRTM (exagero vertical de 50x) da região entre os rios Negro e Manacapuru (AM). A paleo-rede de drenagens, com fluxo para sul, pode ser identificada na porção central da figura, em tons de azul-escuro. Adaptado de Almeia-Filho e Miranda (2007).

A possibilidade de identificar feições de relevo sutis, que seriam mascaradas pela densa cobertura vegetal da região Amazônica em imagens de sensoriamento remoto ótico, foi explorada por Ibanez et al. (2007). A disposição da rede de drenagem de uma área a leste de Manaus (AM) foi utilizada como indicador de padrões estruturais propícios à migração e/ou acumulação de hidrocarbonetos, como falhas e estruturas dômicas (Figura 7). Concluiu-se que análise dos dados morfoestruturais pode direcionar levantamentos geofísicos, e assim reduzir os riscos de campanhas prospectivas em áreas de fronteira exploratória, como as bacias sedimentares paleozóicas da Amazônia.

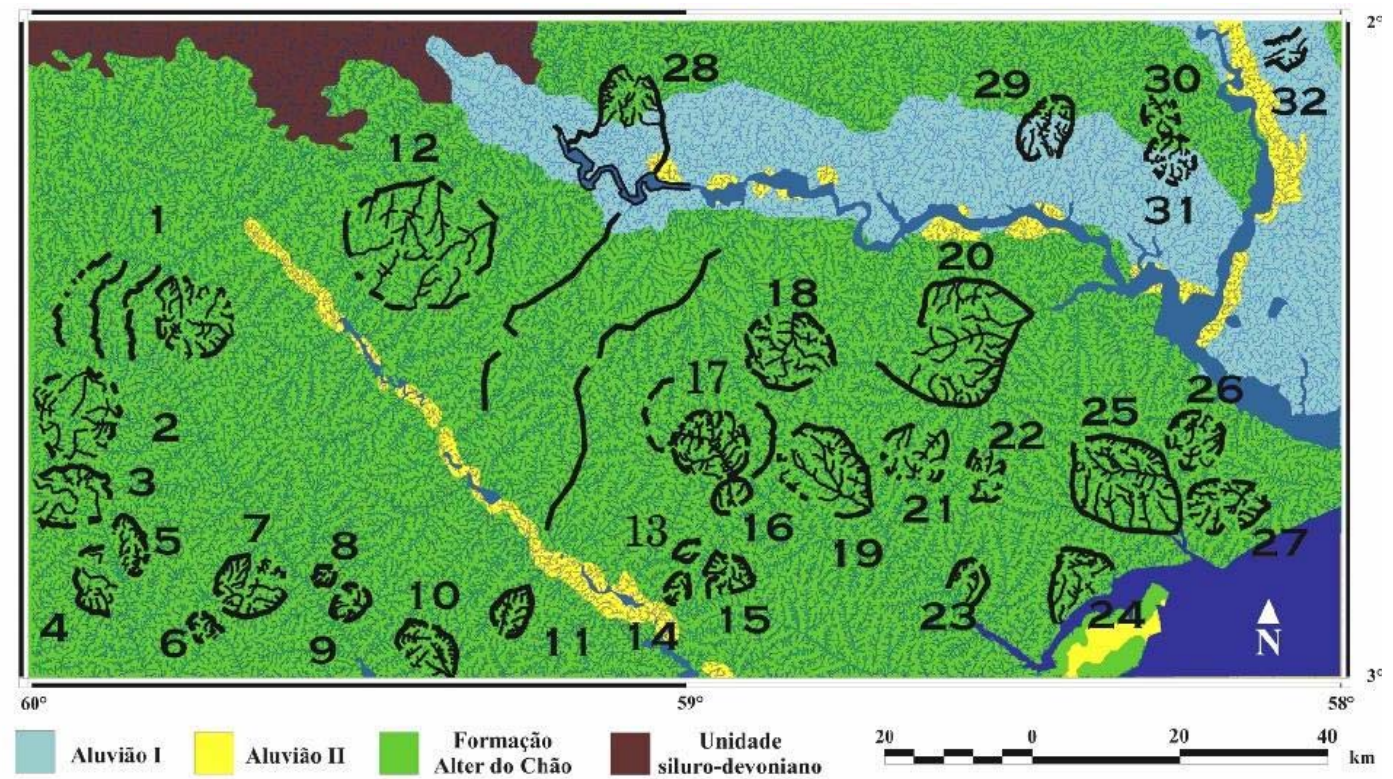

Figura 7 - Mapa geológico com sobreposição da rede de drenagem extraída do modelo SRTM e destaque de anomalias de drenagem identificadas por Ibanez et al. (2007). 


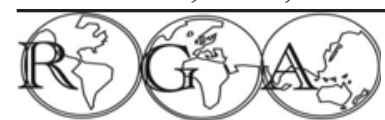

As condições ambientais da região norte brasileira (tal como a presença constante de nuvens) dificultam a aquisição de fotografias aéreas e portanto afetam a qualidade dos mapas topográficos produzidos. Oliveira e Paradella (2008) compararam dados de elevação SRTM, RADARSAT-1 e ASTER com dados altimétricos de precisão levantados em campo para duas áreas no estado do Pará a fim de avaliar sua acurácia e conformidade com o Padrão de Exatidão Cartográfica (PEC) brasileiro [8].

O estudo demonstrou que em uma área de relevo suave (Floresta Nacional do Tapajós - Fig. 8), a acurácia altimétrica dos modelos SRTM (3 segundos de resolução) e RADARSAT-1 (modo Fine) enquadra-se nos requisitos do PEC para cartas Classe A, em escala 1:100.000. Em uma área de relevo montanhoso (Serra dos Carajás), apenas os modelos SRTM eASTER atendem à esses requisitos. Apesar do desempenho ligeiramente superior dos dados ASTER, os autores consideram a dificuldade de se obter imagens sem nuvens e o custo adicional da coleta de pontos de controle para produção dos modelos ASTER-DEM, e sugerem o uso de SRTM como fonte primária de dados de elevação em mapeamentos topográficos de semi-detalhe na região Amazônica.

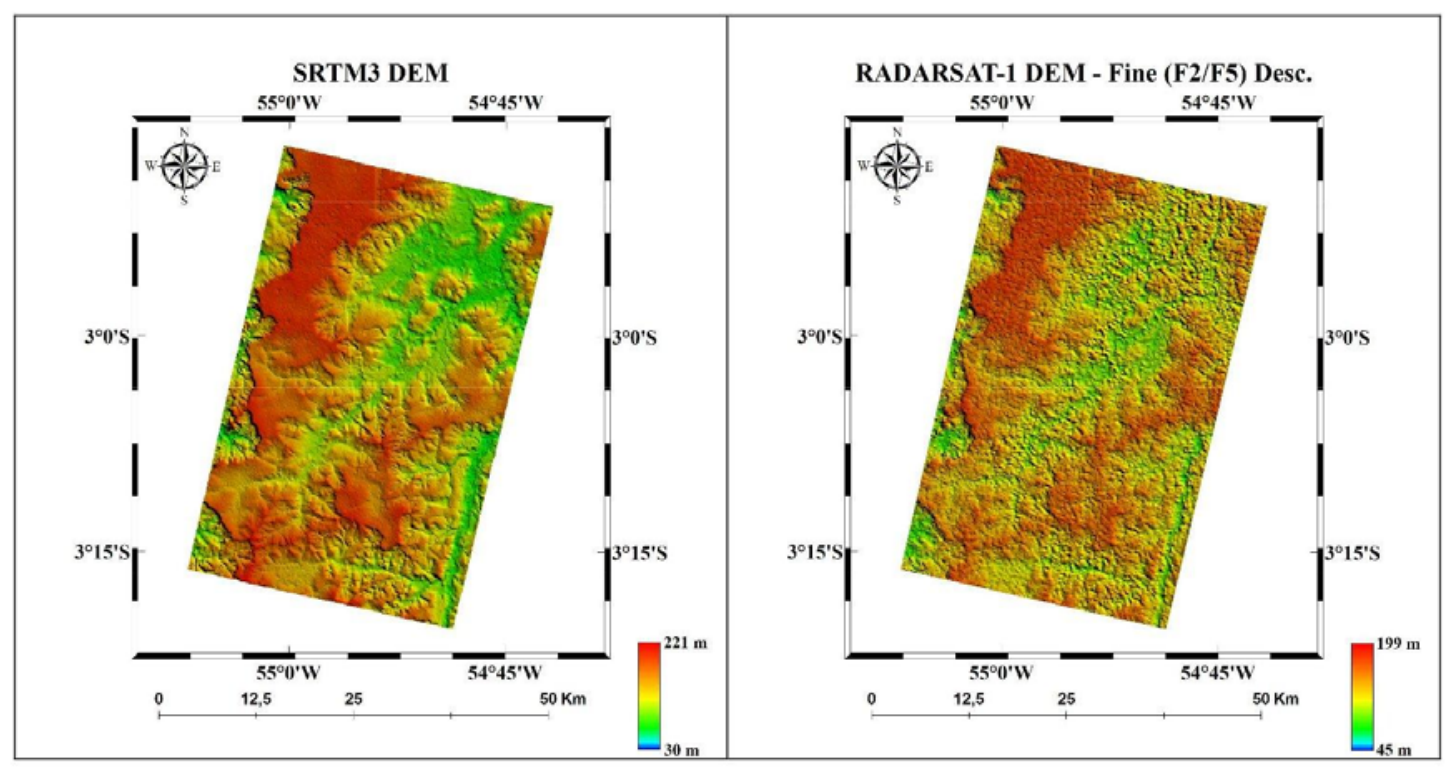

Figura 8 - Modelos de elevação produzidos com SRTM e RADARSAT-1 para a área da Floresta Nacional do Tapajós (Oliveira e Paradella, 2008).

\section{CONCLUSÕES}

Com o advento dos modelos SRTM, abriu-se um amplo leque de possibilidades em estudos geomorfológicos. Entre outras características, modelos de elevação permitem o cálculo de variáveis topográficas com rapidez, a identificação de formas de relevo e de estruturas que seriam mascaradas pela vegetação em imagens ópticas, a visualização a partir de diversos pontos de vista e o cruzamento das informações altimétricas (e variáveis associadas) com dados de campo, de sensoriamento remoto óptico, de geofísica etc. Modelos de abrangência global permitem que análises em escala continental sejam realizadas com um nível de detalhe muito superior ao que se dispunha poucos anos atrás.

No Brasil, boa parte dos estudos publicados concentra-se na região norte, onde as características naturais dificultam a produção de mapas topográficos em escalas de detalhe e semi-detalhe.

Com a crescente popularização dos modelos SRTM e a capacidade dos Sistemas de Informações Geográficas de visualizar/analisar grandes áreas de uma só vez, fator até então limitado pela escala de imagens de satélite, mosaicos de Radar e pelo baixo nível de detalhe dos modelos de elevação regionais, acredita-se que novos dados e interpretações sobre o relevo brasileiro irão surgir, à luz de novos dados e técnicas de processamento. 


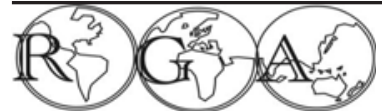

\section{REFERÊNCIAS BIBLIOGRÁFICAS}

Burrough, P.A. 1987. Spatial aspects of ecological data. In: Data Analysis in Community and Landscape Ecology, Cambridge University Press, p.213-251.

Almeida Filho, R.; Miranda, F.P. 2007. Mega capture of the Rio Negro and formation of the Anavilhanas Archipelago, Central Amazônia, Brazil: Evidences in an SRTM digital elevation model. Remote Sensing of Environment, v.110, p.387392.

Almeida Filho, R.; Moreira, F.R.S.; Beisl, C.H. 2005. The Serra da Cangaiha astrobleme as revealed by ASTER and SRTM orbital data. International Journal of Remote Sensing, v.26, p.833-838.

Bamler, R.; Hartl, P. 1998. Synthetic aperture radar interferometry. Inverse Problems, v.14, p.R1-R54.

Becker, J.J.; Sandwell, D.T. 2007. SRTM30PLUS: Data fusion of SRTM land topography with measured and estimated seafloor topography (Version 3.0).

Carvalho, T.M.; Latrubesse, E.M. 2004. Aplicacão de modelo digitais do terreno (MDT) em análises macrogeomorfológicas: o caso da bacia hidrográfica do Araguaia. Revista Brasileira de Geomorfologia, v.5, p.85-93.

Farr, T.G.; Kobrick, M. 2000. Shuttle Radar Topography Mission produces a wealth of data. EOS (Transactions, American Geophysical Union), v.81, p.583-585.

Farr, T.G.; Rosen, P.A.; Caro, E.; Crippen, R.; Duren, R.; Hensley, S.; Kobrick, M.; Paller, M.; Rodriguez, E.; Roth, L.; Seal, D.; Shaffer, S.; Shimada, J.; Umland, J.; Werner, M.; Oskin, M.; Burbank, D.; Alsdorf, D. 2007. The Shuttle Radar Topography Mission. Review of Geophysics, v.45, RG2004.

Grohmann, C.H.; Riccomini, C.; Alves, F.M. 2007. SRTM-based morphotectonic analysis of the Poços de Caldas Alkaline Massif, southeastern Brazil. Computers \& Geosciences, v.33, p.10-19.

Grohmann, C.H.; Steiner, S.S. 2008. SRTM resample with Short Distance-Low Nugget Kriging. International Symposium on Terrain Analysis and Digital Terrain Modelling. Nanjing, China.

Grohmann, C.H.; Steiner, S.S. 2008. SRTM resample with Short Distance-Low Nugget Kriging. International Journal of Geographical Information Science, no prelo, DOI: 10.1080/13658810701730152.

Guth, P.L. 2006. Geomorphometry from SRTM: Comparison to NED. Photogrammetric Engineering and Remote Sensing, v.72, p.269-278.

Ibanez, D.M.; Almeida Filho, R.; Miranda, F.P. 2007. Uso de dados SRTM no auxílio à pesquisa de hidrocarbonetos na Bacia Sedimentar do Amazonas. Anais do XIII Simpósio Brasileiro de Sensoriamento Remoto, Florianópolis, p.20152022.

Jakobsson, M.; Cherkis, N.Z.; Woodward, J.; Macnab, R.; Coakley, B. 2000. New grid of Arctic bathymetry aids scientists and mapmakers. EOS (Transactions, American Geophysical Union), v.81, p.89,93,96.

Kahle, A.B.; Palluconi, F.D.; Hook, S.J.; Realmuto, V.J.; Bothwell, G. 1991. The Advanced Spaceborne Thermal Emission and Reflectance Radiometer (ASTER). International Journal of Imaging Systems and Technology, v.3, p.144-156.

Miranda, E.E. 2005. Brasil em Relevo. Embrapa Monitoramento por Satélite, Campinas.

Oliveira, C.G.; Paradella, W.R. 2008. An assessment of the altimetric information derived from spacebourne SAR (RADARSAT-1, SRTM3) and optical (ASTER) data for cartographic application in the Amazon region. Sensors, v.8, p.3819-3829. 


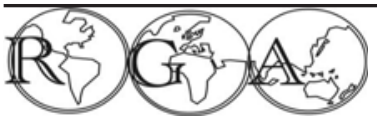

Rabus, B.; Eineder, M.; Roth, R.; Bamler, R. 2003. The Shuttle Radar Topography Mission—a new class of digital elevation models acquired by spaceborne radar. ISPRS Journal of Photogrammetry \& Remote Sensing, v.57, p.241-262.

Rodriguez, E.; Morris, C.H.; Belz, J.E. 2006. A global assessment of the SRTM performance. Photogrammetric Engineering and Remote Sensing, v.72, p.249-260.

Rossetti, D.F.; Valeriano, M.M.; Thales, M. 2007. An Abandoned Estuary Within Marajó Island: Implications for Late Quaternary Paleogeography of Northern Brazil. Estuaries and Coasts, v.30, p.813-826.

Smith, W.H.F.; Sandwell, D.T. 1997. Global seafloor topography from satellite altimetry and ship depth soundings. Science, v.277, p.1957-1962.

Steiner, S.S. 2007. Aquisicão e processamento de dados morfométricos derivados do modelo digital de elevacão SRTM. Dissertação de Mestrado. Instituto de Geociências, Universidade de São Paulo, São Paulo.

Valeriano, M.M. 2004. Modelos digitais de elevacão de microbacias elaborados com krigagem. Serviço de Informação e Documentação (SID), INPE, INPE-9364-RPQ/736, 54pp.

Valeriano, M.M; Kuplich, T.M.; Storino, M.; Amaral, B.D.; Mendes Jr., J.N.; Lima, D.J. 2006. Modeling small watersheds in Brazilian Amazonia with shuttle radar topographic mission-90m data. Computers \& Geosciences, v.32, p.1169-1181.

van Zyl, J.J. 2001. The Shuttle Radar Topography Mission (SRTM): a breakthrough in remote sensing of topography. Acta Astronautica, v.48, p.559-565.

Zebker, H.A. \& Goldstein, R.M. 1986. Topographic mapping from interferometric synthetic aperture radar observations. Journal of Geophysical Research, v.91, p.4993-4999.

\section{REFERENCIAS - INTERNET}

$1-\mathrm{http}: / /$ seamless.usgs.gov

2 - ftp://e0srp01u.ecs.nasa.gov/srtm

3 - http://www.relevobr.cnpm.embrapa.br

4 - http://topex.ucsd.edu/WWW html/srtm30 plus.html

5 - http://edcdaac.usgs.gov/gtopo30/gtopo30.asp

6 - http://ocean-ridge.ldeo.columbia.edu/general/html/home.html

7 - http://www.ngdc.noaa.gov/mgg/coastal/coastal.html

8 - http://www.concar.ibge.gov.br/indexf7a0.html?q=node/41 